\title{
Optimal Closed-loop Control of Timed Event Graphs in Dioids
}

\author{
C. A. Maia L. Hardouin R. Santos-Mendes $\quad$ B. Cottenceau
}

\begin{abstract}
This paper deals with the model-reference control of timed event graphs using the dioid algebra and the residuation theory. It proposes a control structure based on a precompensator and a feedback controller to improve the controlled system performance. It is shown that this approach always leads to an optimal behavior of the closed-loop system. An example is given to illustrate the proposed approach.
\end{abstract}

Keywords : Discrete event dynamic systems, Timed Petri nets, (max, +) algebra, dioid, Control, Just-in-Time.

\section{INTRODUCTION}

Discrete Event Systems (DES) appear in many applications in manufacturing, computer and communication systems and are often described by the Petri Net formalism (see [9]). Timed Event Graphs (TEG) are Timed Petri Nets in which all places have single upstream and single downstream transitions and appropriately model DES characterized by delay and synchronization phenomena. TEG can be described by linear equations in the dioid algebra formulation ([1], [4], [8]) and this fact has permitted many important achievements on the control of DES modelled by

C. A. Maia is with Departamento de Engenharia Elétrica, Universidade Federal de Minas Gerais, Brazil. On leave at Laboratoire d'Ingénierie des Systèmes Automatisés, Université d'Angers, France. Grant from CAPES, Brazil. E-mail: maia@cpdee.ufmg.br

L. Hardouin is with Laboratoire d'Ingénierie des Systèmes Automatisés, Université d'Angers, France . E-mail: laurent.hardouin@istia.univ-angers.fr

R. Santos-Mendes is with Faculdade de Engenharia Elétrica e de Computação, Universidade Estadual de Campinas, Brazil. E-mail: rafael@dca.fee.unicamp.br

B. Cottenceau is with Laboratoire d'Ingénierie des Systèmes Automatisés, Université d'Angers, France . E-mail: bertrand.cottenceau@istia.univ-angers.fr 
TEG, as detailed in [3], [6] , [7], [11] and [12]. TEG control problems are usually stated in a Justin-Time context, where the design goal is to minimize stocks while guaranteeing performance (e.g. throughput). One possible approach for the control of TEG is the model-reference technique in which a given model (written in dioid formalism) describes the desired performance limits and the design goal is achieved through the calculation of a precompensator or of a feedback controller [7], [11]. The techniques based on feedback control, although favoring stability, are limited in the sense that the reference model must satisfy certain restrictive conditions. Those based on precompensation can guarantee performance for any reference model, but not stability (for the concept of stability in TEG, see [5]). The present paper proposes a new technique for the design of controllers based on the simultaneous calculation of a precompensator and a feedback controller. The main advantage of the approach is that it achieves optimality regarding stocks while guaranteeing optimal compliance with any prescribed reference model. In addition sufficient conditions for stabilization of the system are established.

The paper is organized as follows. Section II introduces some algebraic tools concerning the Dioid and Residuation theories and their applications to TEG. Section III introduces some control results and develops the proposed control structure and section IV shows an application to TEG stabilization with an illustrative example. A conclusion is given in section $\mathrm{V}$.

\section{LINEAR SySTEMS THEORY FOR TEG USING DIOID THEORY}

A dioid $\mathcal{D}$ is an algebraic structure with two internal operations denoted by $\oplus$ and $\otimes$. The operation $\oplus$ is associative, commutative and idempotent, that is, $a \oplus a=a$. The operation $\otimes$ is associative (but not necessarily commutative) and distributive at left and at right with respect to $\oplus$. The neutral elements of $\oplus$ and $\otimes$ are represented by $\varepsilon$ and $e$ respectively, and $\varepsilon$ is absorbing for $\otimes(\forall a \in \mathcal{D}, \varepsilon \otimes a=a \otimes \varepsilon=\varepsilon)$. In a dioid, a partial order relation is defined by $a \succeq b$ iff $a=a \oplus b$ and $x \wedge y$ denotes the greatest lower bound between $x$ and $y$. A dioid $\mathcal{D}$ is said to be complete if it is closed for infinite $\oplus$-sums and if $\otimes$ distributes over infinite $\oplus$-sums. Most of the time the symbol $\otimes$ will be omitted as in conventional algebra, moreover, $a^{i}=a \otimes a^{i-1}$ and $a^{0}=e$.

Theorem 1 ( [1], th. 4.75): The implicit equation $x=a x \oplus b$ defined over a complete dioid $\mathcal{D}$, admits $x=a^{*} b$ as least solution, where $a^{*}=\bigoplus_{i \in \mathbb{N}} a^{i}$ (Kleene star operator). 
TEG control problems, stated in a just-in-time context, usually involves the inversion of isotone mappings ${ }^{1}$, that is, one must find $x$ such that $f(x)=y$ (where $f$ is isotone). Residuation Theory [2] deals with such problems stated in partially ordered sets and is based on the following concepts. First, the subsolution set is defined as $\{x \mid f(x) \preceq y\}$. The residuated mapping is defined as follows.

Definition 1 (Residual and residuated mapping): An isotone mapping $f: \mathcal{D} \rightarrow \mathcal{E}$, where $\mathcal{D}$ and $\mathcal{E}$ are partially ordered sets, is a residuated mapping if for all $y \in \mathcal{E}$ there exists a greatest subsolution for the equation $f(x)=y$ (hereafter denoted $f^{\sharp}(y)$ ). The mapping $f^{\sharp}$ is called the residual of $f$.

The mappings $L_{a}: x \mapsto a \otimes x$ and $R_{a}: x \mapsto x \otimes a$ defined over a complete dioid $\mathcal{D}$ are both residuated ([1], p. 181). Their residuals are isotone mappings denoted respectively by $L_{a}^{\sharp}(x)=a \phi x$ and $R_{a}^{\sharp}(x)=x \phi a$. Some useful dioid formulæ involving these residuals are given below.

$$
\begin{aligned}
a(a ф x) & \preceq x \\
a(a ф(a x)) & =a x \\
a ф a & =(a ф a)^{*} \\
\left(a^{*}\right)^{2} & =a^{*}
\end{aligned}
$$

A trajectory of a TEG transition $x$ is a firing date sequence $\{x(k)\} \in \mathbb{Z}$. For each increasing sequence $\{x(k)\}$, it is possible to define the transformation $x(\gamma)=\bigoplus_{k \in \mathbb{Z}} x(k) \gamma^{k}$ where $\gamma$ is a backward shift operator in event domain (that is $y(\gamma)=\gamma x(\gamma) \Leftrightarrow\{y(k)\}=\{x(k-1)\}$, see [1], p. 228). This transformation is analogous to the $Z$-transform used in discrete-time classical control theory and the formal series $x(\gamma)$ is a synthetic representation of the trajectory $x(k)$. The set of the formal series in $\gamma$ is denoted by $\overline{\mathbb{Z}}_{\max } \llbracket \gamma \rrbracket$ and constitutes a dioid. For MIMO TEG, vectors $U \in\left(\overline{\mathbb{Z}}_{\max } \llbracket \gamma \rrbracket\right)^{p}$ and $Y \in\left(\overline{\mathbb{Z}}_{\max } \llbracket \gamma \rrbracket\right)^{m}$ will respectively represent the input and output trajectories of the TEG. These trajectories can be related ([1], p. 243) by the equation $Y=H U$, where $H \in\left(\overline{\mathbb{Z}}_{\max } \llbracket \gamma \rrbracket\right)^{m \times p}$ is called the transfer matrix of the TEG. Entries of matrix $H$ are

\footnotetext{
${ }^{1} f$ is isotone mapping if it preserves order, that is, $a \preceq b \Longrightarrow f(a) \preceq f(b)$.
} 
periodic series ([1], p. 260) in the dioid $\overline{\mathbb{Z}}_{\max } \llbracket \gamma \rrbracket$, usually represented by ${ }^{2} p(\gamma) \oplus q(\gamma)\left(\tau \gamma^{\nu}\right)^{*}$. The asymptotic slope of a periodic series $s=p(\gamma) \oplus q(\gamma)\left(\tau \gamma^{\nu}\right)^{*}$ denoted $\sigma_{\infty}(s)$ is defined as the $\operatorname{ratio}^{3} \sigma_{\infty}(s)=\frac{\nu}{\tau}$.

Theorem 2 ([8], p. 196): Let $s_{1}$ and $s_{2}$ be two periodic series such that $\nu_{1}, \nu_{2} \neq 0$ and $\tau_{1}, \tau_{2} \neq$ 0 , then

$$
\begin{aligned}
\sigma_{\infty}\left(s_{1} \oplus s_{2}\right) & =\min \left(\sigma_{\infty}\left(s_{1}\right), \sigma_{\infty}\left(s_{2}\right)\right), \\
\sigma_{\infty}\left(s_{1} \otimes s_{2}\right) & =\min \left(\sigma_{\infty}\left(s_{1}\right), \sigma_{\infty}\left(s_{2}\right)\right), \\
\sigma_{\infty}\left(s_{1} \wedge s_{2}\right) & =\max \left(\sigma_{\infty}\left(s_{1}\right), \sigma_{\infty}\left(s_{2}\right)\right) .
\end{aligned}
$$

If $\sigma_{\infty}\left(s_{1}\right) \leq \sigma_{\infty}\left(s_{2}\right)$ then $\sigma_{\infty}\left(s_{2} \nmid s_{1}\right)=\sigma_{\infty}\left(s_{1}\right)$, else $s_{2} \nmid s_{1}=\varepsilon$.

\section{Control Method}

The control method proposed herein is based on the Just-in-Time strategy and on the model reference approach [7] and is described as follows. Let $H \in\left(\overline{\mathbb{Z}}_{\max } \llbracket \gamma \rrbracket\right)^{m \times p}$ be the transfer matrix of the plant and $G_{r e f} \in\left(\overline{\mathbb{Z}}_{\max } \llbracket \gamma \rrbracket\right)^{m \times p}$ be the reference model, i.e., the desired transfer matrix for the controlled system. The precompensation problem is solved by finding the greatest precompensator $P$ such that $H P \preceq G_{r e f}$. The optimal solution, denoted by $P_{o p}$, is given by

$$
P_{o p}=H ф G_{r e f} .
$$

This means that, for a given external input ${ }^{4} V \in\left(\overline{\mathbb{Z}}_{\max } \llbracket \gamma \rrbracket\right)^{p}$, the input variable, given by $U=P V$, will be maximal. In fact, for any $P$ such that $H P \preceq G_{r e f}, P \preceq P_{o p}$, therefore the isotony property assures that $U=P V \preceq P_{o p} V$.

In the feedback control context, the closed-loop transfer matrix between $Y$ and $V$, for a given feedback controller $F$, is given by $H(F H)^{*}$. Therefore the problem is solved by finding the greatest $F$ such that $H(F H)^{*} \preceq G_{r e f}$.

${ }^{2} p(\gamma)=\bigoplus_{i=0}^{n-1} p_{i} \gamma^{i}, p_{i} \in \mathbb{N}$, is a polynomial that represents the transient and $q(\gamma)=\bigoplus_{j=0}^{\nu-1} q_{j} \gamma^{j}, q_{i} \in \mathbb{N}$, is a polynomial that represents a pattern which is repeated each $\tau$ time units and each $\nu$ firings of the transition.

${ }^{3}$ Asymptotic slope in a manufacturing context can be viewed as the production rate of the system. The ratio $\sigma_{\infty}(s)$ is calculated in the conventional algebra.

${ }^{4}$ In a Just-in-Time context, $V$ represents the available catering of raw material and $U$ represents the allowance of the raw material into the system. 
This problem can be solved via residuation theory if some restrictions are imposed on the reference model. The following result is due to [7].

Proposition 1: Let $H \in\left(\overline{\mathbb{Z}}_{\max } \llbracket \gamma \rrbracket\right)^{m \times p}$ be the transfer function of a TEG. For every reference model $G_{r e f}$ such that $G_{r e f}=R^{*} H$ or $G_{r e f}=H S^{*}\left(\right.$ where $R \in\left(\overline{\mathbb{Z}}_{\max } \llbracket \gamma \rrbracket\right)^{m \times m}$ and $S \in$ $\left.\left(\overline{\mathbb{Z}}_{\max } \llbracket \gamma \rrbracket\right)^{p \times p}\right)$ there exists a greatest feedback controller $F \in\left(\overline{\mathbb{Z}}_{\max } \llbracket \gamma \rrbracket\right)^{p \times m}$ such that the transfer function of the closed-loop system is less than or equal to $G_{r e f}$. The greatest feedback controller is:

$$
F_{o p}=H \phi G_{r e f} \phi H .
$$

In this strategy the transfer function between $U$ and $V$ is $(F H)^{*}$.

Property 1: If $H(F H)^{*} \preceq G_{r e f}$, for given $H$ and $G_{r e f}$, then $(F H)^{*} \preceq P_{o p}=H \nmid G_{r e f}$.

The proof comes from the fact that $a x \preceq b \Leftrightarrow x \preceq a \oint b$. This property means that the transfer function between $U$ and $V$ for the optimal open-loop strategy is always greater than or equal to the one obtained for any feasible feedback strategy.

The model-reference control scheme proposed in this paper is a generalization of the two strategies described above, that is, it uses a precompensator and a feedback controller together. The two main advantages of this strategy compared with the simple feedback is that it always leads to an optimal control signal which is equal to $P_{o p} V$ and there is no restriction concerning the reference model choice. Fig. 1 illustrates the approach.

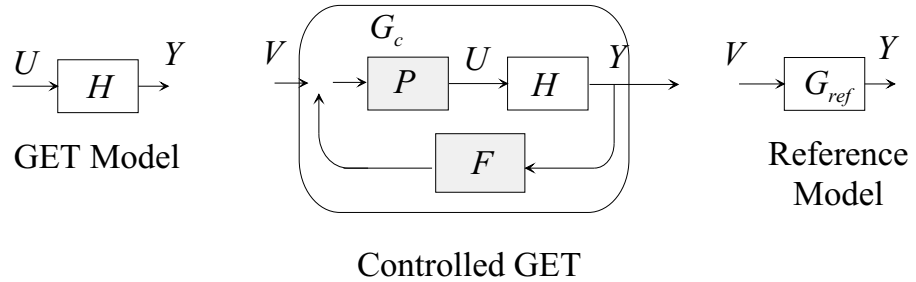

Fig. 1. Proposed Control Structure

By using theorem 1, one can obtain the closed-loop equations which relate $U, V$ and $Y$ :

$$
\begin{aligned}
Y=G_{c} V=(H P F)^{*} H P V & =H P(F H P)^{*} V ; \\
U=G_{u v} V & =P(F H P)^{*} V ;
\end{aligned}
$$


where $G_{c}=(H P F)^{*} H P$ and $G_{u v}=P(F H P)^{*}$ represent closed-loop transfer functions between $Y$ and $V$ and between $U$ and $V$ respectively. The problem can be stated as follows. Given $a$ TEG, what are the controller matrices $P$ and $F$ which assure the greatest transfer function between $U$ and $V$, i.e. $G_{u v}$, such that $G_{c} \preceq G_{r e f}$ ? Again, considering the Just-in-Time context, one seeks the controller which satisfies the reference specification $G_{c} \preceq G_{r e f}$ while delaying as much as possible the entrance of products to be processed. Formally, the problem can be stated as follows:

$$
\begin{gathered}
\bigoplus_{P, F} G_{u v}(P, F) \\
\text { s.t. } G_{c}=H P(F H P)^{*} \preceq G_{r e f} .
\end{gathered}
$$

This statement shows that $P=[\varepsilon]_{p \times p}$ is always a subsolution to the problem independently of the choice of $F$, meaning that the subsolution set is not empty. Furthermore, it is easy to notice that the strategies using exclusively a precompensator (by setting $F=[\varepsilon]_{p \times m}$ ) or exclusively a feedback controller (by setting $P=I_{p \times p}$, where $I_{p \times p}$ is the identity matrix in dioid) are particular cases of the above problem.

Proposition 2: For the proposed control scheme shown in Fig. 1, the three following inequalities are equivalent:

$$
\begin{aligned}
H P(F H P)^{*} & \preceq G_{r e f} \\
P(F H P)^{*} & \preceq H \phi G_{r e f} \\
H P(F H P)^{*} & \preceq H\left(H ф G_{r e f}\right)
\end{aligned}
$$

Proof: The relation $H P(F H P)^{*} \preceq G_{r e f} \Rightarrow P(F H P)^{*} \preceq H \nmid G_{r e f}$ comes from the residuation definition. $P(F H P)^{*} \preceq H \downarrow G_{r e f} \Rightarrow H P(F H P)^{*} \preceq H\left(H \nmid G_{r e f}\right)$ comes from the isotony of $\otimes$. Finally $H P(F H P)^{*} \preceq H\left(H \downarrow G_{r e f}\right) \Rightarrow H P(F H P)^{*} \preceq G_{r e f}$ is due to inequality (1), concluding the proof.

Lemma 1: Let $\mathcal{S}_{a}=\left\{x \mid x^{*} \preceq a^{*}\right\}$ be a subset of the complete dioid $\mathcal{D}$ where $a \in \mathcal{D}$. Then $\mathcal{S}_{a}=\left\{x \mid x \preceq a^{*}\right\}$ and as a consequence the greatest element of $\mathbb{S}_{a}$ is $a^{*}$.

Proof: It is sufficient to show the equivalence $x^{*} \preceq a^{*} \Leftrightarrow x \preceq a^{*}$. If $x^{*} \preceq a^{*}$ then $x^{*}=e \oplus x \oplus x^{2} \oplus \ldots \preceq a^{*}$ so $x \preceq a^{*}$. On the other hand, if $x \preceq a^{*}$, then $x^{2} \preceq x a^{*} \preceq a^{*} a^{*} \preceq a^{*}$ 
because of the isotony of the multiplication and equation (4). By induction, one obtains $\forall i \geq 1$, $x^{i} \preceq a^{*}$, which leads to $x^{*}=e \oplus x \oplus x^{2} \oplus \ldots \preceq a^{*}$.

Lemma 2: A solution to problem 8 must satisfy $P \preceq G_{u v} \preceq H \phi G_{r e f}$.

Proof: Straightforward from the problem definition and from the observation that $G_{u v}=$ $P(F H P)^{*}=P \oplus \bigoplus_{i=1}^{\infty} P(F H P)^{i}$ which implies that $P \preceq G_{u v}$.

Proposition 3: A solution to the optimization problem proposed in (8) is given by:

$$
\begin{aligned}
& P_{o p}=H \phi G_{r e f} . \\
& F_{o p}=\left(H P_{o p}\right) \phi\left(H P_{o p}\right) \phi\left(H P_{o p}\right) .
\end{aligned}
$$

Proof: From lemma 2, $G_{u v}$ is maximum (it is equal to the upper bound) if $P=H \nmid G_{r e f}$ and $F=\varepsilon$. So the greatest $F$ for this value of $P$ is given by the greatest subsolution of inequality $P_{o p}\left(F H P_{o p}\right)^{*} \preceq H \nmid G_{r e f}$, which in turn (by proposition 2 ) is equivalent to $H P_{o p}\left(F H P_{o p}\right)^{*} \preceq$ $H\left(H \nmid G_{r e f}\right)=H P_{o p}$. Moreover, from the residuation definition this inequality is equivalent to $\left(F H P_{o p}\right)^{*} \preceq\left(H P_{o p}\right) \nmid\left(H P_{o p}\right)$. Equation (3) yields $\left(\left(H P_{o p}\right) \uparrow\left(H P_{o p}\right)\right)^{*}=\left(H P_{o p}\right) \phi\left(H P_{o p}\right)$ then, thanks to lemma $1, F H P_{o p} \preceq\left(H P_{o p}\right) \oint\left(H P_{o p}\right)$. Finally, by solving this last inequality one obtains $F_{o p}=\left(H P_{o p}\right) \phi\left(H P_{o p}\right) \phi\left(H P_{o p}\right)$.

One must observe here that unlike the approach depicted in proposition 1, the proposed approach does not restrict the reference model choice.

Property 2: The solution given by proposition 3 assures that $G_{u v}=H \nmid G_{r e f}$ and $G_{c}=H P_{o p}$. This property follows directly from proposition 3 , lemma 2 and from the observation that $G_{c}=$ $H G_{u v}$ given by equations (6) and 7. It means that the proposed solution always assures that greatest closed-loop transfer functions $G_{u v}$ and $G_{c}$ are equal to their upper bounds, that is, $P_{o p}$ and $H P_{o p}$ respectively.

Property 3: If there exists a matrix $D$ such that $G_{r e f}=H D$ then the optimal solution for the proposed control structure leads to $G_{c}=G_{r e f}$. This condition means that the closed-loop system effectively matches the reference model.

Proof: According to property 2, it is sufficient to show that $H P_{o p}=G_{r e f}$ under the given conditions. Equation (9) gives $P_{o p}=H \nmid(H D)$. Therefore $H P_{o p}=H(H \nmid(H D))$ which is equal to $H D$ by equation (2).

If the goal is to preserve the system impulse response, that is $G_{r e f}=H$, an optimal solution is achieved if $P_{o p}=H ф H$ and $F_{o p}=H ф H \phi H$. Again one uses the fact that $a(a ф a)=a$, thanks to 
equation (2).

\section{AppliCATION: TEG STABILIZATION}

Consider the TEG depicted in Fig. 2 as an illustrative example. It represents a workshop with 3 machines $\left(M_{1}\right.$ to $\left.M_{3}\right)$ and its input-output equation is given by

$$
y=\left[\begin{array}{ll}
7(3 \gamma)^{*} & 9(4 \gamma)^{*}
\end{array}\right] u \text {. }
$$

It is important to observe that this TEG is unstable since production rates of machine $M_{1}$ and $M_{2}$ are different. This means that the number of tokens in the place between $M_{1}$ and $M_{3}$ can be unbounded for some inputs. However it is known that a TEG is stable under certain conditions, e.g., the TEG is strongly connected [5]. By definition ([1], p. 305) an event graph is structurally controllable if every internal transition can be reached by a path from at least one input transition. It is structurally observable if, from every internal transition, there exists a path to at least one output transition. These concepts allow the following.

Proposition 4 (TEG Stabilization): Let $H$ be a transfer function of a TEG structurally controllable and observable and denote $\sigma_{\infty}(H)=\operatorname{Min}_{1 \leq i \leq m, 1 \leq j \leq p}\left(\sigma_{\infty}\left(H_{i j}\right)\right)$. If $G_{r e f}$ is such that $\sigma_{\infty}\left(\left[G_{r e f}\right]_{i j}\right)=\lambda \leq \sigma_{\infty}(H) \forall i \in[1, m]$ and $\forall j \in[1, p]$, then equations (9) and (10) lead to optimal controllers, $P_{o p}$ and $F_{o p}$, which assure stability of the closed-loop system.

Proof: If a TEG is structurally controllable and observable, in every row and in every column of $H$ there exists a non null entry. According to matrix residuation([1], p.198), $\left[P_{o p}\right]_{i j}=$ $\bigwedge_{k=1}^{m} H_{k j} \oint\left[G_{r e f}\right]_{k i}, \forall i, j \in[1, p]$. Since $\sigma_{\infty}\left(\left[G_{r e f}\right]_{k i}\right)=\lambda \leq \sigma_{\infty}(H)$ then by theorem 2 , $\sigma_{\infty}\left(\left[P_{o p}\right]_{i j}\right)=\lambda, \forall i, j \in[1, p]$. This result means that matrix $P_{o p}$ is full and all its entries have the same asymptotic slope. Furthermore $\sigma_{\infty}\left(\left[H P_{o p}\right]_{i j}\right)=\lambda \leq \sigma_{\infty}(H) \forall i \in[1, m]$ and $\forall j \in[1, p]$. Therefore the TEG resulting from a composition of $P$ and $H$ is also structurally controllable and observable. Similarly from equation (10), one gets $\sigma_{\infty}\left(\left[F_{o p}\right]_{i j}\right)=\lambda \forall i \in[1, p]$ and $\forall j \in[1, m]$. So every entry $\left[F_{o p}\right]_{i j} \neq \varepsilon$ have the same asymptotic slope which implies that each output of the system is connected to each input. As a result, since the system is structurally controllable and observable, the closed-loop system is strongly connected. Therefore, as remarked before [5], it is stable. 
For the example modelled by equation $(11), \sigma_{\infty}(H)=\frac{1}{4}$. If the reference model is chosen as $^{5} G_{r e f}=\left[10(4 \gamma)^{*} 10(4 \gamma)^{*}\right]$ all the conditions required in the theorem 4 are fulfilled. The optimal solutions given by equations (9) and (10) are respectively ${ }^{6} P_{o p}=(4 \gamma)^{*}\left(\begin{array}{ll}3 & 3 \\ 1 & 1\end{array}\right)$ and $F_{o p}=(4 \gamma)^{*}\left(\begin{array}{l}-10 \\ -10\end{array}\right)$. This feedback is not causal because there are negative coefficients in matrix entries meaning a negative date for the transition firings (see [1], p.259, for a strict definition of causality in dioid). However the canonical injection ${ }^{7}$ from the set of causal elements of $\overline{\mathbb{Z}}_{\max } \llbracket \gamma \rrbracket$ (denoted $\overline{\mathbb{Z}}_{\text {max }}^{+} \llbracket \gamma \rrbracket$ ) in $\overline{\mathbb{Z}}_{\text {max }} \llbracket \gamma \rrbracket$ is also residuated (see [6] for details). Its residual is denoted $\operatorname{Pr}_{+}$ and its computation for all $s \in \overline{\mathbb{Z}}_{\max } \llbracket \gamma \rrbracket$ is given by

$$
\operatorname{Pr}_{+}\left(\bigoplus_{k \in \mathbb{Z}} s(k) \gamma^{k}\right)=\bigoplus_{k \in \mathbb{Z}} s_{+}(k) \gamma^{k}
$$

where $s_{+}(k)=\left\{\begin{array}{l}s(k) \text { if }(k, s(k)) \geq(0,0), \\ \varepsilon \text { otherwise. }\end{array}\right.$

In practice, this result means that the negative coefficients of the series can be simply eliminated. In [6] it is demonstrated, in a slightly different context, that the application of operator $\operatorname{Pr}_{+}$to the solution of the proposed problem preserves optimality. This result can be extended to the problem stated in this paper, that is, $\left(\operatorname{Pr}_{+}\left(P_{o p}\right), \operatorname{Pr}_{+}\left(F_{o p}\right)\right)$ is an optimal solution to the problem stated in equation (8) when $P$ and $F$ are restricted to the causal set. Actually, since $G_{u v} \preceq H \phi G_{r e f}$ a causal upper bound for $G_{u v}$ is $\operatorname{Pr}_{+}\left(H \phi G_{r e f}\right)$. As a result, if $P=\operatorname{Pr}_{+}\left(H \phi G_{r e f}\right)$ and $F=\varepsilon$ then $G_{u v}$ is maximum. By using an argument similar to that presented in proposition 3, one can demonstrate that the maximal causal feedback is given by $\operatorname{Pr}_{+}\left(\left(H P_{o p}\right) \phi\left(H P_{o p}\right) \phi\left(H P_{o p}\right)\right)$. Therefore the greatest causal feedback for the example is $F_{o p_{+}}=\operatorname{Pr}_{+}\left(F_{o p}\right)=2 \gamma^{3}(4 \gamma)^{*}\left(\begin{array}{l}e \\ e\end{array}\right)$. Fig. 2 shows one realization of the controlled workshop system.

\footnotetext{
${ }^{5}$ This reference does not satisfy the conditions of the proposition 1 . Indeed $G_{r e f} \neq R^{*} H$ and $G_{r e f} \neq H S^{*}$.

${ }^{6}$ Softwares to handle dioid algebra using Scilab language can be downloaded from the sites [13].

${ }^{7} I: V \mapsto W$ with $\mathrm{V} \subset W, x \mapsto x$.
} 


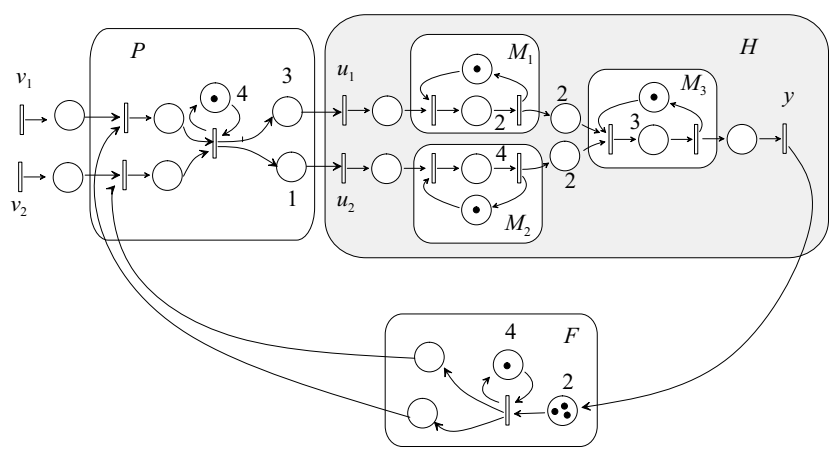

Fig. 2. Controlled Workshop System

\section{CONCLUSION}

This paper presents a new method for the TEG model-reference control in the context of dioid theory. A structure based on precompensation as well as on feedback assures an optimal solution to the just-in-time control problem together with optimal compliance with any model reference. The results herein presented generalize the results found in the literature based exclusively on precompensation or exclusively on feedback control. A main result is the absolute absence of restrictions concerning the choice of the reference model $G_{r e f}$. Moreover, sufficient conditions are derived to guarantee the closed-loop system stabilization and an example illustrates the applicability of the results. The proposed conditions are not necessary and a complete solution for the TEG stabilization problem is yet a concern in the context of TEG control theory. As well, robustness analysis issues remain to be explored in future works, as indicated by [10].

\section{REFERENCES}

[1] F. Baccelli, G. Cohen, G.J. Olsder, and J.P. Quadrat. Synchronization and Linearity : An Algebra for Discrete Event Systems. Wiley and Sons, 1992.

[2] T.S. Blyth and M.F. Janowitz. Residuation Theory. Pergamon press, 1972.

[3] J.-L. Boimond, J.-L. Ferrier. Internal Model Control and Max-Algebra: Controller Design. IEEE Trans. on Automat. Contr., 41(3):457-461, 1996.

[4] G. Cohen, P. Moller, J.P. Quadrat, and M. Viot. Algebraic Tools for the Performance Evaluation of Discrete Event Systems. IEEE Proc.: Spec. Issue on Disc. Event Syst., 77(1):39-58, Jan. 1989.

[5] C. Commault. Feedback Stabilization of Some Event Graph Models. IEEE Trans. on Automat. Contr., 43(10):1419-1423, Oct. 1998.

[6] B. Cottenceau, L. Hardouin, J.-L. Boimond, and J.-L. Ferrier. Synthesis of Greatest Linear Feedback for TEG in Dioid. IEEE Trans. on Automat. Contr., 44(6):1258-1262, 1999. 
[7] B. Cottenceau, L. Hardouin, J.-L. Boimond, and J.-L. Ferrier. Model Reference Control for Timed Event Graphs in Dioid. Automatica, 37:1451-1458 2001.

[8] S. Gaubert. Théorie des Systèmes Linéaires dans les Dioïdes. Thèse, École des Mines de Paris, Jul. 1992.

[9] L. Holloway, B. Krogh, and A. Giua. A survey of Petri Net Methods for Controlled Discrete Event Systems. Discrete Event Dynamic Systems: Theory and Applications, 7(2):151-190, 1997.

[10] M. Lhommeau, L. Hardouin, B. Cottenceau and J.-L. Boimond. Sur les correcteurs en boucle fermée dans les dioïdes. Modél. des syst. réact. (MSR'2001), Toulouse, France , 463-478, 2001.

[11] R. Lüders, R. Santos-Mendes. Generalized Multivariable Control of Discrete Event Systems in Dioid. In 6th Int. Workshop on Disc. Event Syst. - WODES'02, Zaragoza, Spain, Oct. 2002.

[12] E. Menguy, J.-L. Boimond, L. Hardouin, and J.-L. Ferrier. Just-in-time Control of Timed Event Graphs Update of Reference Input, Presence of Uncontrollable Input. IEEE Trans. on Automat. Contr., 45(11):2155-2158, Nov. 2000.

[13] SW2001. Software Tools for Manipulating Periodic Series. http://www.istia-angers.fr/ hardouin/outils.html, http://amadeus.inria.fr/gaubert/papers/max.html, http://www-rocq.inria.fr/scilab/quadrat/quadrat.html, 2001. 